\title{
Correction to: Early Adolescent Skills for Emotions (EASE) intervention for the treatment of psychological distress in adolescents: study protocol for randomised controlled trials in Lebanon and Jordan
}

Felicity L. Brown ${ }^{1}$, Frederik Steen ${ }^{1}$, Karine Taha ${ }^{2}$, May Aoun², Richard A. Bryant ${ }^{3}$, Mark J. D. Jordans ${ }^{1,4^{*}}$, Aiysha Malik ${ }^{5}$, Mark van Ommeren ${ }^{5}$, Adnan Abualhaija ${ }^{6}$, Ibrahim Said Agel ${ }^{6}$, Maha Ghatasheh ${ }^{6}$, Rand Habashneh ${ }^{6}$, Marit Sijbrandij ${ }^{7}$, Rabih El Chammay ${ }^{8,9}$, Sarah Watts ${ }^{5}$, Aemal Akhtar ${ }^{3,7}$ and on behalf of the STRENGTHS-consortium

Correction to: Trials (2019) 20:545

https://doi.org/10.1186/s13063-019-3654-3

Following publication of the original article [1], we have been notified that Fig. 1 was published with the track changes. In this Correction the incorrect and correct Fig. 1 are shown.

The original article has been corrected.

\begin{abstract}
Author details
${ }^{1}$ Research and Development Department, War Child Holland, Amsterdam,

The Netherlands. ${ }^{2}$ War Child Holland Lebanon Office, Beirut, Lebanon.

${ }^{3}$ School of Psychology, University of New South Wales, Sydney, Australia.

${ }^{4}$ Amsterdam Institute of Social Science Research, University of Amsterdam, Amsterdam, the Netherlands. ${ }^{5}$ Department of Mental Health and Substance Abuse, World Health Organization, Geneva, Switzerland. ${ }^{6}$ Institute for Family Health, Amman, Jordan. ${ }^{7}$ Clinical, Neuro and Developmental Psychology, VU University, Amsterdam, The Netherlands. ${ }^{8}$ Ministry of Public Health, Beirut, Lebanon. ${ }^{9}$ Department of Psychiatry, Faculty of Medicine, Saint Joseph University, Beirut, Lebanon.
\end{abstract}

Published online: 29 October 2019

\section{Reference}

1. Brown FL, et al. Early Adolescent Skills for Emotions (EASE) intervention for the treatment of psychological distress in adolescents: study protocol for randomised controlled trials in Lebanon and Jordan. Trials. 2019;20:545. https://doi.org/10.1186/s13063-019-3654-3.

\footnotetext{
*Correspondence: mark.jordans@warchild.nl

${ }^{1}$ Research and Development Department, War Child Holland, Amsterdam, The Netherlands

${ }^{4}$ Amsterdam Institute of Social Science Research, University of Amsterdam, Amsterdam, the Netherlands

Full list of author information is available at the end of the article
}

(c) The Author(s). 2019 Open Access This article is distributed under the terms of the Creative Commons Attribution 4.0 International License (http://creativecommons.org/licenses/by/4.0/), which permits unrestricted use, distribution, and reproduction in any medium, provided you give appropriate credit to the original author(s) and the source, provide a link to the Creative Commons license, and indicate if changes were made. The Creative Commons Public Domain Dedication waiver (http://creativecommons.org/publicdomain/zero/1.0/) applies to the data made available in this article, unless otherwise stated. 
Originally Fig. 1 has been published as:

\begin{tabular}{|c|c|c|c|c|c|c|c|}
\hline \multirow[b]{7}{*}{ Measure } & & Formattod & \\
\hline & & & & & & Formatted & \\
\hline & & & & & & Formatted & \\
\hline & \multicolumn{5}{|c|}{ Study Period } & Formatted & \\
\hline & Enrolment & Baseling & Post- & 3 month & 12 month & Formatted & \\
\hline & cirominent & Dasentive & & fFollow-up & ffollow-up & Formatted & \\
\hline \multirow{2}{*}{\multicolumn{6}{|c|}{$\begin{array}{l}\text { Measure } \\
\text { ENROLMENT: }\end{array}$}} & Formatted & \\
\hline & & & & & & Formatted & \\
\hline \multirow{3}{*}{$\begin{array}{l}\text { Eligibility screen } \\
\text { Informed consent } \\
\text { Allocation }\end{array}$} & $\mathrm{x}$ & & & & & Formattod & \\
\hline & $x$ & & & & & Formatted & ] \\
\hline & & $x$ & & & & Formattod & \\
\hline \multirow{4}{*}{$\begin{array}{l}\text { INTERVENTIONS: } \\
\text { EASE }\end{array}$} & & & & & & Formattod & \\
\hline & & . & & & & Formatted & $\bar{r}$ \\
\hline & & & & & & Formatted & \\
\hline & & & & & & Formattod & \\
\hline & & & & & & Formattod & \\
\hline $\begin{array}{l}\text { ASSESSMENTS: } \\
\text { Demographics }\end{array}$ & & $x$ & & & & Formattod & I \\
\hline $\begin{array}{l}\text { Demographics } \\
\text { Child-reported psychological distress }\end{array}$ & $x^{1}$ & $\frac{x}{x}$ & $x$ & $x$ & $x$ & Formattod & \\
\hline Child-reported depression symptoms & & $\hat{x}$ & $\hat{x}$ & $\hat{x}$ & $\frac{x}{x}$ & Formattod & \\
\hline Child-reported post-traumatic stress symptoms & & $\frac{x}{x}$ & $\frac{x}{x}$ & $\frac{x}{x}$ & $\frac{x}{x}$ & Formattod & 1 \\
\hline Child-reported wellbeing & & $\hat{x}$ & $\hat{x}$ & $\hat{x}$ & $\frac{1}{x}$ & Formattod & \\
\hline Child-reported impairment in daily functioning & & $x$ & $x$ & $x$ & $\hat{x}$ & Formattod & \\
\hline Child coping strategr use? & & & & & & Formattod & 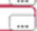 \\
\hline Psychological Sense of School Membership ${ }^{3}$ & & $x$ & $x$ & $x$ & $x$ & Formattod & $\vec{a}$ \\
\hline Parent-reported child psychological distress & & $x$ & $x$ & $x$ & $x$ & Formattod & \\
\hline Parenting practices & & $x$ & $x$ & $\hat{x}$ & $\hat{x}$ & Formattod & \\
\hline Parent mental health & & $x$ & $x$ & $x$ & $x$ & Formattod & \\
\hline Parent coping strategy use $\mathrm{e}^{2}$ & & $\mathrm{x}$ & $\mathrm{x}$ & $\mathrm{x}$ & $\mathrm{x}$ & Formatted & \\
\hline Child exposure to potentially traumatic events & & $x$ & & & & Formatted & \\
\hline \multirow{2}{*}{$\begin{array}{l}\text { Attendance at sessions } \\
\text { Contamination }\end{array}$} & & & $x$ & & & Formatted & \\
\hline & & & $x$ & $x$ & & Formattod & \\
\hline & Formattod & \\
\hline & & & & & & Formattod & \\
\hline
\end{tabular}


The correct figure is:

\begin{tabular}{|c|c|c|c|c|c|}
\hline \multirow[b]{3}{*}{ Measure } & \multicolumn{5}{|c|}{ Study Period } \\
\hline & Enrolment & Baseline & $\begin{array}{c}\text { Post- } \\
\text { intervention }\end{array}$ & $\begin{array}{l}3 \text { month } \\
\text { follow-up }\end{array}$ & $\begin{array}{l}12 \text { month } \\
\text { follow-up }\end{array}$ \\
\hline & & TO & $\mathrm{T} 1_{\text {(8 weeks) }}$ & $T 2_{\text {(20weeks) }}$ & T3 (60 weeks) \\
\hline \multicolumn{6}{|l|}{ ENROLMENT: } \\
\hline \multirow{3}{*}{$\begin{array}{l}\text { Eligibility screen } \\
\text { Informed consent } \\
\text { Allocation }\end{array}$} & $\mathrm{x}$ & & & & \\
\hline & $\mathrm{x}$ & & & & \\
\hline & & $\mathrm{X}$ & & & \\
\hline \multicolumn{6}{|l|}{ INTERVENTIONS: } \\
\hline \multicolumn{6}{|l|}{ EASE } \\
\hline \multicolumn{6}{|l|}{ ETAU } \\
\hline \multicolumn{6}{|l|}{ ASSESSMENTS: } \\
\hline \multicolumn{6}{|l|}{ Demographics } \\
\hline \multirow{4}{*}{$\begin{array}{l}\text { Child-reported psychological distress } \\
\text { Child-reported depression symptoms } \\
\text { Child-reported post-traumatic stress symptoms } \\
\text { Child-reported wellbeing }\end{array}$} & $\mathrm{x}^{1}$ & $x$ & $x$ & $x$ & $x$ \\
\hline & & $x$ & $x$ & $x$ & $x$ \\
\hline & & $x$ & $x$ & $x$ & $x$ \\
\hline & & $x$ & $\mathrm{x}$ & $x$ & $\mathrm{x}$ \\
\hline \multirow{2}{*}{$\begin{array}{l}\text { Child-reported impairment in daily functioning } \\
\text { Child coping strategy use } \\
\text { Psychological Sense of School Membership }\end{array}$} & & $x$ & $x$ & $x$ & $x$ \\
\hline & & $\mathrm{x}$ & $\mathrm{x}$ & $x$ & $x$ \\
\hline $\begin{array}{l}\text { Parent-reported child psychological distress } \\
\text { Parenting practices }\end{array}$ & & $x$ & $x$ & $x$ & $x$ \\
\hline Parenting practices & & $\mathrm{x}$ & $\mathrm{x}$ & $\mathrm{x}$ & $\mathrm{x}$ \\
\hline \multirow{2}{*}{$\begin{array}{l}\text { Parent mental health } \\
\text { Parent coping strategy use }{ }^{2}\end{array}$} & & $x$ & $x$ & $x$ & $x$ \\
\hline & & $\mathrm{x}$ & $x$ & $x$ & $\mathrm{x}$ \\
\hline \multirow{2}{*}{$\begin{array}{l}\text { Child exposure to potentially traumatic events } \\
\text { Attendance at sessions }\end{array}$} & & $x$ & & & \\
\hline & & & $x$ & & \\
\hline Contamination ${ }^{2}$ & & & $\mathrm{x}$ & $x$ & \\
\hline
\end{tabular}

Fig. 2 Standard Protocol Items Recommendations for Interventional Trials (SPIRIT): Schedule of enrolment, interventions, and assessments for trials of EASE. ${ }^{1} 17$-item screener. ${ }^{2}$ Lebanon only. ${ }^{3}$ Jordan only 